\title{
Norois
}

Environnement, aménagement, société

\section{Le simulateur DAHU : une plateforme de modélisation des activités humaines en zone côtière}

The DAHU simulation platform: modelling human activities in coastal zone

Cyril Tissot, Matthieu Le Tixerant et Mathias Rouan

\section{(2) OpenEdition}

Journals

Édition électronique

URL : https://journals.openedition.org/norois/455

DOI : $10.4000 /$ norois. 455

ISBN : 978-2-7535-1544-4

ISSN : 1760-8546

\section{Éditeur}

Presses universitaires de Rennes

\section{Édition imprimée}

Date de publication : 1 septembre 2005

Pagination : 125-135

ISBN : 978-2-7535-0218-5

ISSN : 0029-182X

\section{Référence électronique}

Cyril Tissot, Matthieu Le Tixerant et Mathias Rouan, « Le simulateur DAHU : une plateforme de modélisation des activités humaines en zone côtière », Norois [En ligne], 196 | 2005/3, mis en ligne le 05 août 2008, consulté le 13 janvier 2022. URL : http://journals.openedition.org/norois/455 ; DOI : https://doi.org/10.4000/norois.455

Ce document a été généré automatiquement le 13 janvier 2022.

(C) Tous droits réservés 


\section{Le simulateur DAHU : une plateforme de modélisation des activités humaines en zone côtière}

The DAHU simulation platform: modelling human activities in coastal zone

Cyril Tissot, Matthieu Le Tixerant et Mathias Rouan

\section{NOTE DE L'ÉDITEUR}

Cet article a été reçu le 3 mars 2005 et définitivement accepté le 6 octobre 2005.

1 À l'interface entre l'atmosphère, la terre et l'océan, les zones côtières sont des systèmes complexes particulièrement riches, très convoités par les sociétés humaines. L'analyse et la compréhension des interactions entre les usages qui s'y développent et l'environnement sont donc un objectif majeur de la recherche pour l'aide au développement durable (Cuq, 2001). La problématique est fondée sur le constat d'une détérioration de la qualité de l'environnement dans les zones côtières, liés notamment au développement d'activités humaines parfois conflictuelles. Les tentatives de la Gestion Intégrée des Zones Côtières ont pour objectif la mise en place d'approches globales afin d'anticiper et de résoudre ces dysfonctionnements (Burbridge, 1998). Dans ce contexte, l'analyse des interactions entre les activités humaines et le milieu et leur observation à long terme apparaissent indispensables (Turner et al., 1996).

Malgré les résultats scientifiques importants obtenus depuis dix ans, on constate toujours une difficulté à développer l'ouverture pluridisciplinaire nécessaire, en particulier en ce qui concerne le lien entre sciences du milieu et sciences humaines (Barnoin, Chardy, 1998). Néanmoins, si l'on se réfère à l'expérience internationale dans ce domaine, il apparaît que les principales avancées concernant la prise en compte des conditions écologiques et humaines se sont appuyées sur des méthodes géomatiques qui ont permis d'établir un lien tangible entre les différents compartiments des systèmes étudiés, de représenter et de modéliser les interactions entre les dynamiques 
écologiques et sociales (Bousquet et al., 1996 ; Cuq, 1993 ; Jordão et al., 1996 ; Klemas et al., 1995 ; Staljanssens, 1998).

3 La modélisation des interactions entre nature et société est un sujet qui est abordé depuis plusieurs années par les chercheurs en Sciences humaines sous l'aspect des interactions entre les changements du climat et les systèmes économiques (Von Neuman, Debreu, Georgescu-Roegen et Koopmans). Plus récemment, sont apparus des modèles fondés sur la théorie du jeu et l'intelligence artificielle dont l'objectif est de simuler des agents ${ }^{1}$ autonomes et évolutifs en situation d'interaction mutuelle. Cependant, ces systèmes multi-agents ${ }^{2}$ sont principalement employés pour des opérations de simulation opérationnelle ou pré-opérationnelle appliquées à des processus se déroulant en temps quasi-réel, et doivent donc être adaptés à une modélisation intégrant des contraintes spatio-temporelles multi-échelles.

4 L'examen des différentes contributions scientifiques traitant de la modélisation des interactions homme/milieu démontre la difficulté d'intégration des dimensions spatiales et temporelles dans les approches proposées (Gould, 1987 ; Stonebraker et al., 1990 ; Allen, 1991 ; Muxart et al., 1992 ; Snodgrass, 1992 ; Cheylan et al., 1994 ; Claramunt et al., 1999 ; Parent et al., 1999 ; Legay, 2000). La principale limite des développements actuels est inhérente à la variabilité de l'influence anthropique sur les milieux naturels. La dynamique des écosystèmes n'est pas systématiquement synchronisée à l'évolution des activités humaines mais obéit à des cycles beaucoup plus complexes particulièrement difficiles à modéliser. Une telle remarque peut paraître évidente, pourtant la plupart des modèles proposés fonctionne dans des environnements où toutes les entrées/sorties sont considérées comme connues. Ces démarches sont, de ce fait, inapplicables lorsque la spécification du problème est incomplète ou lorsque l'environnement est évolutif, ce qui est le cas des activités anthropiques.

5 Cette contribution vise donc à explorer de nouvelles voies méthodologiques dans la constitution de modèles capables de simuler le déroulement d'activités humaines dans l'espace et dans le temps dans la perspective d'évaluer leurs impacts potentiels sur les milieux concernés. Cette finalité place l'ensemble de la démarche mise en œuvre dans les perspectives des programmes internationaux IGBP (International GeosphereBiosphere Programme) et IHDP (International Human Dimensions Programme on global environmental change) qui cherchent à rendre compte de la complexité des interactions nature/sociétés.

\section{La plate-forme de simulation « Dynamique des Activités Humaines» (DAHU)}

6 La modélisation quantitative repose généralement sur une démarche déterministe qu'il est quasiment impossible d'utiliser pour simuler les processus de décision ou d'adaptation des sociétés humaines aux changements de l'environnement dans lequel elles évoluent.

7 Cependant, entre une telle approche quantitative inopérante sur le long terme et une modélisation qualitative hypothétique des processus globaux d'évolution de la société, il semble possible de développer une voie intermédiaire dans le but d'établir un lien entre ces deux approches. 
8 Cette voie intermédiaire consiste à employer une démarche quasi-déterministe fondée sur la simulation des modes d'utilisation du milieu au moyen d'une plate-forme logicielle à intelligence artificielle distribuée.

9 Cette plate-forme, baptisée Dynamique des Activités HUmaines (DAHU) (Tissot, 2003; Tissot et Cuq, 2004), a pour vocation de simuler le déroulement d'activités anthropiques à fort impact environnemental et d'en étudier les interactions avec l'environnement. Son schéma de construction s'inspire de l'architecture multi-agents du point de vue conceptuel mais présente d'importantes différences dans la formalisation des relations entre les agents et leur espace de vie. En effet, contrairement aux Systèmes MultiAgents (SMA) consacrés à l'analyse des modes d'évolution des paysages à partir de scénarios de production économique (Bousquet et al., 1993; Bonnefoy et al., 2001; Cambier, 1994 ; Sanders et al., 1997), DAHU n'a pas pour but de modéliser l'évolution de l'occupation du sol à long terme mais plutôt de considérer le contexte spatial comme une contrainte de simulation. Par cette approche, le prototype DAHU s'apparente d'avantage à un système à intelligence artificielle distribuée fondé sur le couplage de modèles quantitatifs et qualitatifs au sein d'un Système d'Information Géographique (Tissot et Cuq, 2004).

10 Le modèle DAHU considère l'écosystème étudié comme un potentiel de ressources exploité par les hommes en fonction des techniques dont ils disposent et de leur organisation sociale à une époque donnée. Cette exploitation n'est pas systématiquement associée à un prélèvement, il peut également s'agir d'un usage du territoire propre à une activité spécifique.

11 La construction de ce simulateur repose donc sur la transcription d'un environnement réel complexe en un modèle de réalité ${ }^{3}$ fondé sur la description du déroulement d'un ensemble d'activités humaines dans l'espace et dans le temps (fig. 1). 
Figure 1 : Schéma conceptuel de la plate-forme de simulation DAHU Conceptual diagram of the DAHU simulation platform

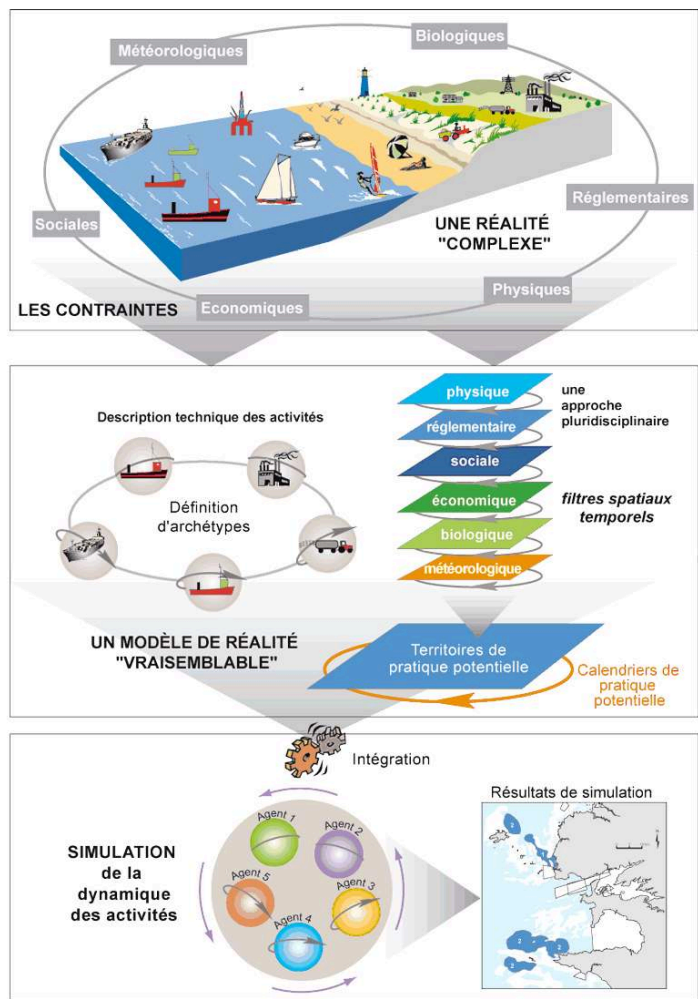

Ce modèle «vraisemblable ${ }^{4}$ » regroupe les conditions d'exercice d'une ou de plusieurs pratiques socio-économiques. Ces informations peuvent être analysées de manière indépendante ou combinée en fonction de leur domaine d'application et de leur durée de validité. L'interprétation de ces différents paramètres donne lieu à l'établissement d'un territoire et d'un calendrier de pratique potentielle pour chaque activité. L'objectif est d'initialiser un espace de modélisation cohérent à partir de l'ensemble des contraintes régissant les pratiques économiques simulées.

La spécificité de ce prototype est donc de baser l'ensemble de la démarche de modélisation sur la description technique d'activités humaines. Chaque activité est représentée par un agent autonome capable de réagir à un environnement vraisemblable préalablement modélisé. Le choix des agents est réalisé à partir d'une typologie détaillée des différentes activités et de leur impact potentiel sur le milieu. Pour contourner les limites inhérentes à une approche non-déterministe, les aspects concernant les pratiques individuelles ne sont pas pris en compte et seules les pratiques ayant atteint un stade d'intensification avancé sont intégrées dans la démarche.

14 Une telle limite s'avère peu contraignante dans une approche environnementale, les activités productivistes ayant généralement le plus fort impact sur le milieu naturel. De plus, cette logique permet de bénéficier de la forte inertie temporelle des activités ${ }^{5}$, ce qui autorise la formalisation d'agents archétypiques représentatifs d'un ou de plusieurs métiers. Il devient dès lors possible de décomposer le cycle de production de manière relativement simple en mettant de côté les aspects concernant les modes individuels de gestion. Tous ces paramètres ont en effet été standardisés par la double influence des avancées techniques et de l'instauration de systèmes productivistes dans la plupart des 
secteurs d'activité. Partant de cette hypothèse, il devient possible d'adjoindre à chacun de ces archétypes ${ }^{6}$ un agent générique autonome représentatif du déroulement typique de l'activité. La modélisation de l'impact potentiel d'une activité (quelle qu'en soit sa nature) est de ce fait intimement conditionnée par l'introduction des contraintes spatio-temporelles dans la chaîne de modélisation.

Sur le plan spatial, il est possible d'établir, pour chaque activité, un Territoire de Pratique Potentielle (TPP) (fig. 1). Il constitue la mise en évidence de zones où est susceptible de se pratiquer l'activité humaine en fonction des contraintes qui conditionnent spatialement son déroulement. Le Territoire de Pratique Potentielle d'une activité est déterminé grâce à la projection spatiale des contraintes de pratique et la superposition des différents filtres spatiaux ainsi obtenus. Pour un site donné, les territoires de pratique peuvent varier, se combiner et se superposer en fonction des activités analysées.

Sur le plan temporel, chaque activité obéit à un cycle de fonctionnement spécifique ajusté à partir de filtres environnementaux, météorologiques, réglementaires et socioéconomiques permettant d'aboutir à la construction d'un Calendrier de Pratique Potentielle (fig. 1). Parallèlement, l'analyse de la variabilité inter-annuelle des composantes du milieu implique de diviser le temps en une série d'intervalles plus ou moins longs en fonction des processus qui leur sont associés. L'ensemble de ces phénomènes n'évoluant pas au même rythme, il apparaît nécessaire de doter les agents d'une unité temporelle commune, compatible dans une perspective d'assemblage avec d'autres modèles. Cette référence a pour principale caractéristique d'être indivisible au sein du modèle, ce que certains auteurs associent à la notion de chronon (Jensen et al., 1994). En conséquence, l'unité généralement choisie correspond au plus petit dénominateur commun à l'ensemble des agents modélisés. Néanmoins, cette règle demande quelques ajustements afin de ne pas générer des situations incohérentes dans le couplage entre les données de forçage et les modèles quantitatifs.

Du point de vue conceptuel, une telle démarche impose un important travail de désagrégation afin d'atteindre ce pas de temps unitaire. Dans le cas des activités humaines, cette décomposition est d'autant plus importante que la plupart des bases de données disponibles résultent d'enquêtes statistiques agrégées à la commune, ne permettant pas de rendre compte de leur variabilité spatio-temporelle.

Afin de contourner cette difficulté, il est envisageable de reconstituer un déroulement typique d'agents possédant un fonctionnement basé sur des processus récurrents. Cette désagrégation temporelle a été réalisée sans tenir compte de l'emprise spatiale des différents éléments intégrés dans le simulateur DAHU. Le temps est donc considéré comme un élément structurant du modèle, chargé de faire le lien entre un agent en perpétuelle évolution et des états de territoire observés à des instants $t$. 


\section{Structuration du simulateur}

Afin de reconstituer une relation spatio-temporelle cohérente entre les modèles d'activités et le territoire dans lequel ils évoluent, la plate-forme de simulation DAHU se compose de trois modules (fig. 2) :

- un pré-processeur dont le rôle est de construire un territoire de pratique potentielle à partir d'une série de filtres réglementaires, socio-économiques et environnementaux, permettant de formaliser l'espace de déroulement d'une activité donnée à un instant $\mathrm{t}$;

- une chaîne de calcul sous contraintes, chargée de simuler le fonctionnement des activités en intégrant différentes variables d'étalonnage et de forçage calculées par le pré-processeur ;

- un post-processeur dédié à la représentation spatiale des résultats de simulation et à la production de couches thématiques multi-échelles (restitution de l'impact d'une série d'activités à un instant t par exemple).

Figure 2 : Structuration de la plate-forme de simulation DAHU Structuring of the DAHU simulation platform

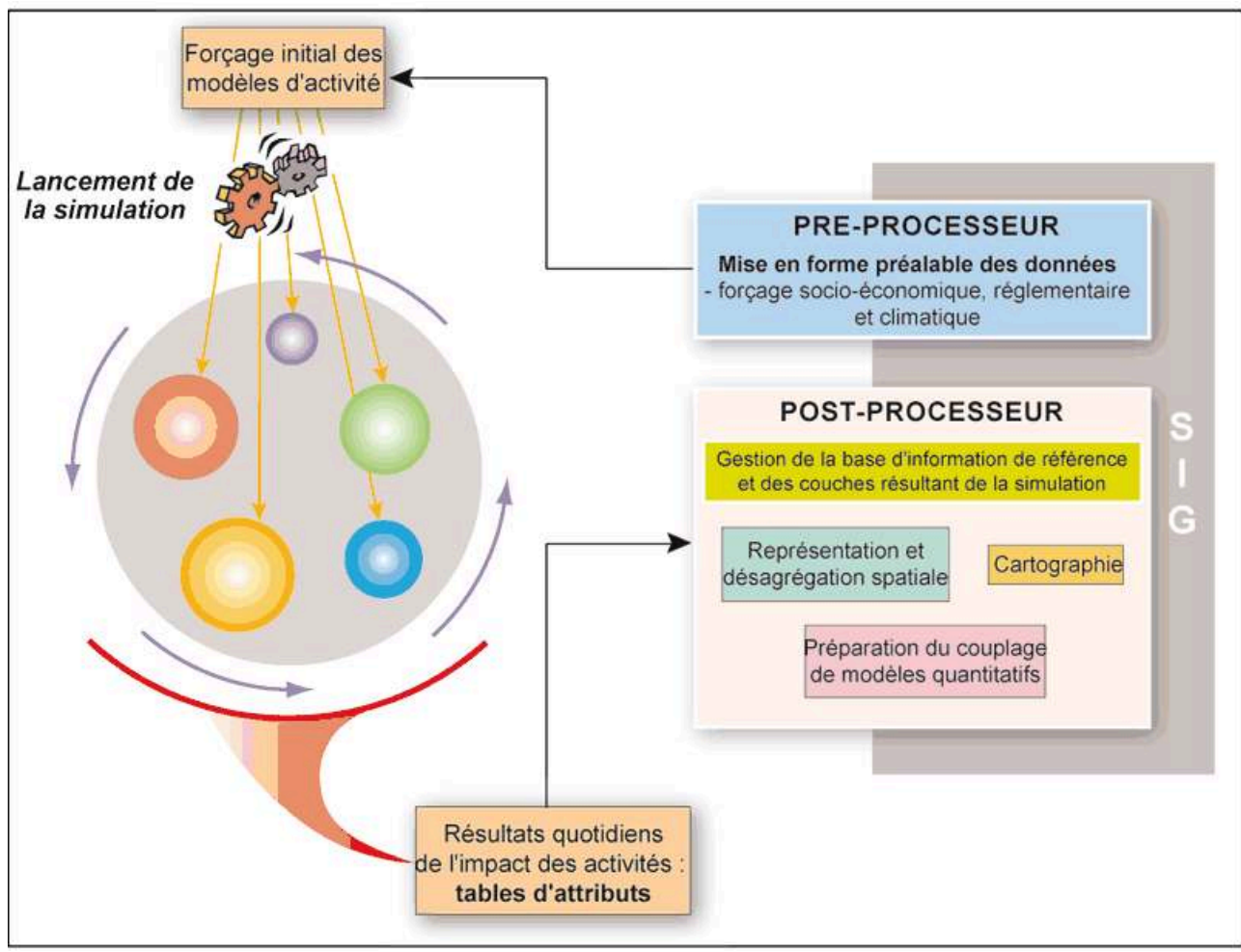

Ces trois éléments font appel à un couplage avec un Système d'Information Géographique (SIG), piloté par le logiciel ArcInfo, chargé de préparer l'ensemble des paramètres nécessaires à la simulation. Le couplage utilisé est de type "Import-export interactif ", la communication DAHU/SIG étant rétroactive. Cette structure permet d'utiliser les sorties de modèle sans formatage préalable et de simuler différents scénarios à partir de forçages spatiaux émanant du logiciel ArcInfo. Ce schéma relationnel est identique pour l'ensemble des facteurs exerçant une pression sur le déroulement des activités. L'avantage de cette méthode réside dans la conservation d'une relation espace/temps cohérente quel que soit le pas de temps choisi. Néanmoins, la complexité des phénomènes décrits mais surtout les interactions entre les facteurs 
physiques et sociaux (paramètres climatiques et cadre juridique par exemple) impliquent une structuration rigoureuse des données.

21 Afin d'optimiser les temps de calcul, le SIG possède donc des fonctions de préprocesseur découplées du simulateur. La communication entre ces deux éléments s'effectue à partir de tables attributaires reliées entre elles par des codes communs. Toutefois, la méthode utilisée par DAHU dépasse le simple échange de données géographiques entre un SIG et un modèle.

Le couplage mis en œuvre tient compte des dimensions spatiale et temporelle des couches d'information. En effet, les Bases d'Information à Référence Spatiale (BDRS) pilotées par le SIG intègrent une description des propriétés temporelles des objets géographiques. Cette particularité permet d'adapter le type de données intégrées au modèle en fonction de la période et du pas de temps choisis.

\section{Intégration des agents et déroulement des simulations}

Le schéma d'assemblage de la chaîne de calcul pilotée par la plate-forme DAHU est directement dérivé des diagrammes de classes réalisés à l'issue de l'étape de spécification de chacun des modèles d'activités. Le formalisme Unified Modeling Language (UML) a été utilisé et enrichi de stétéotypes spatiaux pour l'ensemble des applications développées (fig. 3).

Figure 3 : Application du schéma organisationnel du simulateur DAHU aux activités d'élevage agricole, d'après Tissot C., 2003

Organizational diagram application of the DAHU simulator to cattle breeding activities

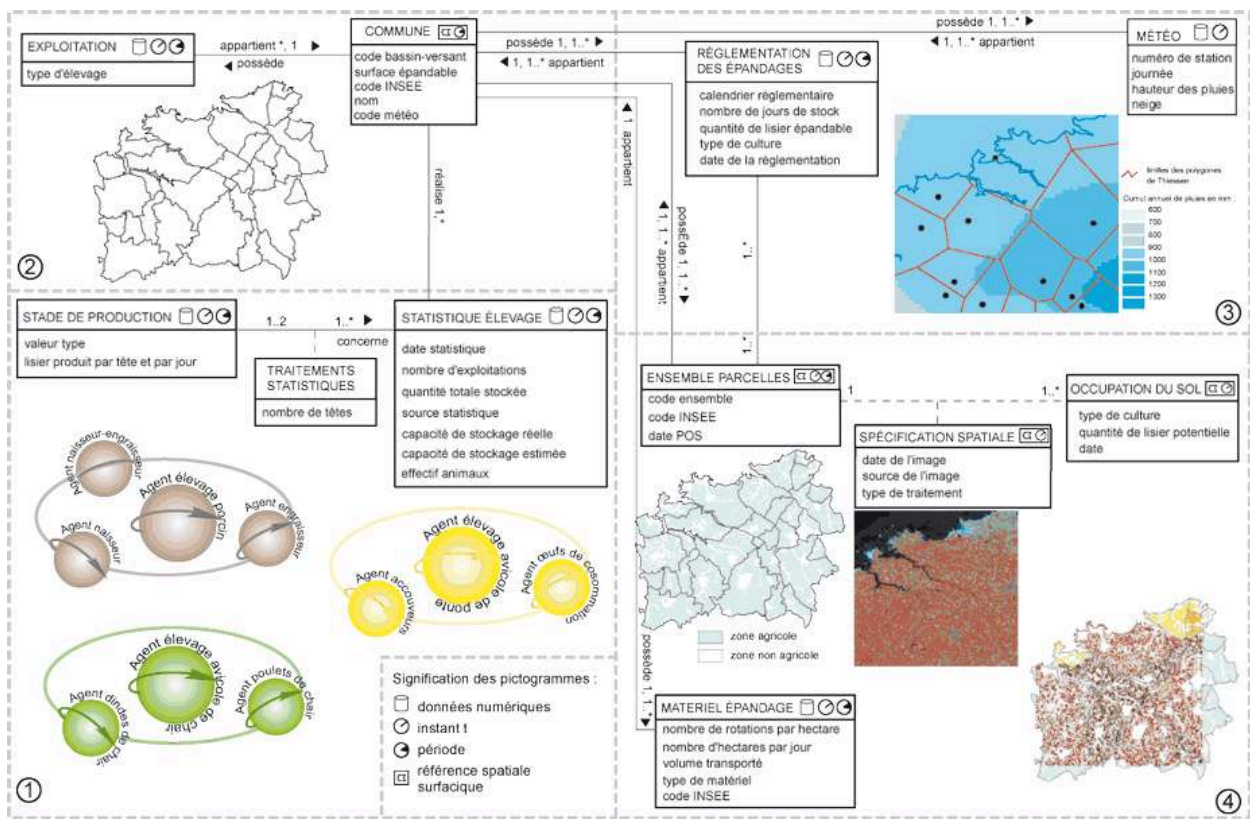

L'objectif de cette démarche est de prendre en compte les propriétés topologiques des objets géographiques intégrés au sein du pré-processeur de manière à structurer l'information à différents niveaux scalaires. Il s'agit d'introduire un ensemble de clefs d'agrégation et de désagrégation spatiale au sein du modèle afin de conserver un rapport espace/temps cohérent entre les agents et les variables de forçage. 
25 La figure 3 propose une application de cette méthode au niveau des activités d'élevage agricole. Le diagramme de classes présenté intègre des informations relatives à la nature des données spatiales et temporelles associées à chacune des classes. Il correspond en ce sens à une interprétation géographique du formalisme UML. Ce mode de représentation s'avère pertinent dans l'optique de disposer d'un dictionnaire de données intégrant des métadonnées relatives aux propriétés spatio-temporelles des objets géographiques administrés dans le pré-processeur. L'ensemble des agents et des procédures de calcul a été implémenté en langage orienté objet $\left(\mathrm{C}_{++}^{+}\right)$, ce référentiel de programmation étant bien adapté à la simulation de processus autonomes et offrant un bon compromis entre portabilité et optimisation des temps de calcul (Rouan, 2001). Les différentes tâches sont exécutées chronologiquement en fonction des règles de production propres à chacune des activités.

26 Les simulations sont étalonnées sur un pas de temps journalier correspondant au plus petit dénominateur commun à l'ensemble des processus modélisés. Autrement dit, chaque boucle de calcul est parcourue quotidiennement durant toute la période de simulation. Cette référence temporelle est relativement contraignante car elle impose de gérer simultanément plusieurs événements possédant des durées d'exécution dissemblables.

27 Afin de conserver une bonne cohérence dans l'exécution des algorithmes, chaque agent est identifié à un thread ${ }^{7}$ intégrant des données techniques propres au fonctionnement de l'activité simulée et des contraintes spatio-temporelles calculées par le préprocesseur (fig. 4). À partir de ces variables, la chaîne de calcul crée un ensemble d'archétypes permettant de décrire techniquement le déroulement des activités correspondantes et initialise un territoire de pratique potentiel afin de déterminer l'impact des ces activités. 
Figure 4 : Principe de fonctionnement du simulateur DAHU Operation principle of the DAHU simulator

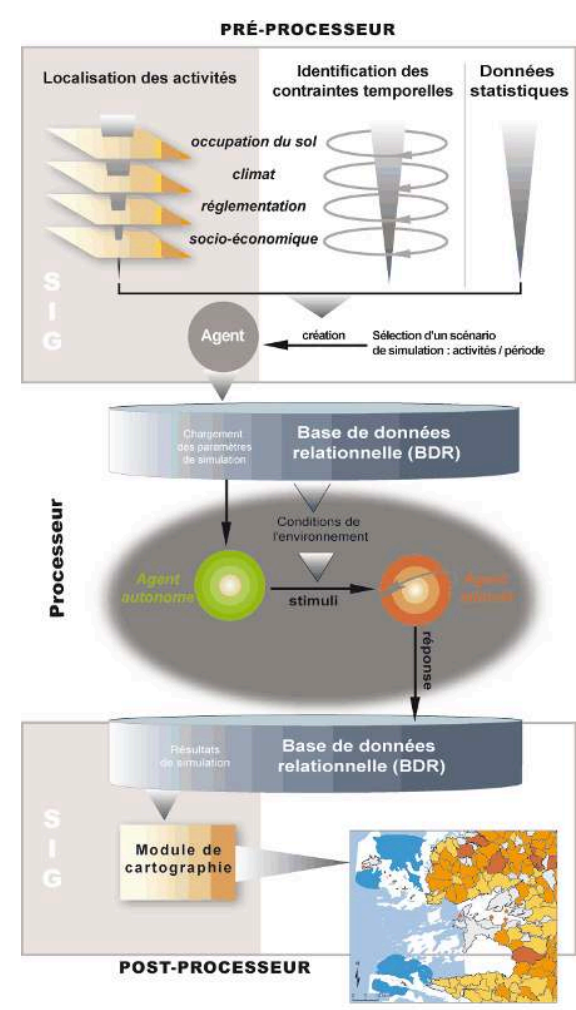

Enfin, le simulateur calcule la production de chacune des activités et leurs impacts potentiels sur le(s) milieu(x) concerné(s).

Ce système permet de gérer l'ensemble des traitements au sein d'un même processus et ainsi de donner une totale autonomie de fonctionnement aux agents simulés. Cette opération est également essentielle au forçage des agents, ceux-ci étant stimulés par des paramètres spatio-temporels spécifiques à leur localisation géographique.

La réponse des agents se présente sous la forme de tables attributaires qui contiennent l'ensemble des variables permettant de quantifier et de qualifier le déroulement des activités simulées. Ces tables sont intégrées dans une base de données relationnelle et sont structurées en référence aux couches d'information géographique correspondant aux territoires de pratique potentielle.

31 Cette architecture permet une exploitation directe des résultats de simulation par le SIG couplé au simulateur mais également la formalisation d'un nouveau jeux de données de forçage directement intégrable via la pré-processeur.

\section{Les Modules Activités Littorales (MAL) et Activités Marines (MAM)}

De manière à tenir compte des spécificités du déroulement des activités sur les espaces terrestres et marins, la plate-forme DAHU a été dotée de deux modules :

- un Module Activités Littorales (MAL) pour les activités se déroulant en zones terrestre et infra-littorale ;

- un Module Activités Marines (MAM) pour les activités se déroulant en mer côtière. 

l'impact des activités d'élevage intensif à l'échelle du département du Finistère (Tissot et al., 2001 ; Tissot, 2003 ; Tissot et Cuq, 2004 ; Tissot et al., 2004). Pour être en mesure de quantifier et de qualifier les épandages résultant des activités d'élevage intensif, il est essentiel de connaître précisément leur origine et leur fréquence d'utilisation. Cette démarche ne concerne donc pas seulement l'évaluation des quantités de lisiers et fumiers produits aux cours des différents cycles d'élevage mais également leur utilisation à des fins fertilisantes. De manière à disposer au sein du même modèle des activités présentes dans et à l'émissaire des bassins versants côtiers, ce module traite également des cultures marines (Palleau, 2004).

Le Module Activités Marines est spécifiquement dédié aux activités humaines se déroulant en mer côtière telles que la pêche, la navigation maritime, l'extraction de matériaux... L'objectif de ce modèle est de fournir une description quotidienne du déroulement des principales activités marines à différentes échelles spatio-temporelles (Le Tixerant et al., 2003; Le Tixerant, 2004 ; Le Tixerant, 2005). Les résultats obtenus permettent d'aider à évaluer les impacts potentiels de ces activités sur les écosystèmes marins et les interactions spatio-temporelles entre activités, sources de conflits potentiels.

\section{Conclusion}

Le développement de la plate-forme de simulation DAHU s'inscrit dans un projet de recherche visant à modéliser les changements à long terme de l'environnement côtier sous l'influence des activités humaines.

Du point de vue méthodologique, la plate-forme DAHU repose sur la transcription d'un environnement complexe en un modèle de réalité fondé sur la description archétypique du déroulement d'activités humaines dans l'espace et dans le temps. Cette démarche est relativement innovante compte tenu de la difficulté de développer une approche déterministe dans le domaine des sciences humaines (Cuq, 2001). L'intérêt de cette méthode réside dans la possibilité de modéliser une activité à l'aide d'un agent autonome possédant l'ensemble de ses caractéristiques de fonctionnement. Ce schéma de construction s'inspire de l'architecture développée dans les Systèmes Multi-Agents (SMA) du point de vue conceptuel mais présente d'importantes différences dans la formalisation des relations entre les agents et leur espace de vie. L'un des principaux apports du prototype DAHU est de considérer l'espace comme une contrainte de simulation et non comme un élément constitutif du modèle. Un tel système permet de fournir une description «hors-sol » d'une activité donnée en s'affranchissant de toute perturbation liée au particularisme des conditions locales de son implantation. Il est ainsi envisageable de reconstituer le cycle de fonctionnement de la plupart des activités possédant un système de production basé sur des processus récurrents sans relation directe avec l'espace concerné. Cette démarche permet de découpler le fonctionnement des activités modélisées avec leur environnement extérieur et ainsi de combler une grande partie des limites inhérentes à l'absence de synchronisation entre les dynamiques sociales et les dynamiques naturelles.

En l'état actuel de son développement, la plate-forme DAHU permet de simuler à la fois des activités terrestres (agriculture intensive) et marines (pêche, navigation,

Norois, 196 | 2005/3 
aquaculture), grâce notamment à l'utilisation de données communes (zone d'étude, données de référence...). À moyen terme, DAHU a pour vocation de refléter l'organisation et le fonctionnement de la zone côtière dans sa globalité et sa diversité afin de pouvoir décrire les différents états de ce territoire, ainsi que les causes de son évolution. Le simulateur évoluerait ainsi vers un véritable système prospectif permettant d'aboutir à une vision globale du déroulement et de l'impact des activités humaines en zone côtière (Cuq, 2001 ; Tissot et al., 2004).

\section{BIBLIOGRAPHIE}

ALLEN (J. F.), 1991. - « Time and time again: the many ways to represent time », International Journal of Intelligent Systems, $\mathrm{n}^{\circ}$ 6/4, p. 341-355.

BARNOUIN (B.), CHARDY (P.), 1998. - Editorial de la Lettre PIGB-PMRC, 7 p.

BONNEFoy (J.-L.), BousQuet (F.), ROUCHIER (J.) 2001. - « Modélisation d'une interaction individus, espace et société par les systèmes multi-agents : pâture en forêt virtuelle », L'espace géographique, $\mathrm{n}^{\circ} 1$, p. 13-25.

Bousquet (F.), Cambier (C.), Mullon (C.), Morand (P.), Quensière (J.), PaVÉ (A.), 1993. - « Simulating the interaction between a society and a renewable resource ", Journal of biological systems, $\mathrm{n}^{\circ} 1, \mathrm{p}$. 199-214.

BOUSQUet (F.), BARRETEAU (O.), WEBER (J.), 1996. - « Systèmes multi-agents et couplage de modèles biophysiques et socio-économique ", Couplage de modèles en agriculture, Montpellier, CIRAD (pagination inconnue).

BURBRIDGE (P. R.), 1998. - « Provocateur's comments on transforming theories of integrated coastal management into practice ", Third European Marine Science and Technology Conference, Lisbonne, p. 175-190.

CAMBIER (C.), 1994. - SIMDELTA, Un système multi-agents pour simuler la pêche sur le delta central du Niger, Thèse de l'université de Paris IV, 197 p.

CheYlan (J.-P.), LARDon (S.), MATHIAN (H.), SANDERs (L.), 1994. - « Les problématiques liées au temps dans les SIG », Revue Internationale de Géomatique, $n^{\circ} 4$ 3/4, p. 287-305.

Claramunt (C.), Parent (C.), Spaccapietra (S.), Theriault (M.), 1999. - « Database Modelling for Environmental and Land Use Changes », dans OPENSHAW (s.), GEERTMAN (s.), STILLWELl (J.) (ed.), Geographical Information and Planning : European Perspectives, Springer-Verlag, p. 173-194.

CUQ (F.), 1993. - « L'utilisation des systèmes d'information géographique pour l'analyse du fonctionnement des écosystèmes ", Les études pluridisciplinaires et les systèmes d'information environnementale, ICALPE, MAB-UNESCO/ministère de l'Environnement, p. 63-68.

-, 2001. - « Analyse des interactions entre les actions humaines et le milieu littoral : une approche discrète de l'impact des activités anthropiques », CoastGIS ‘01, Université Saint Mary's, Halifax, $10 \mathrm{p}$. 
CUQ (F.), BOURSier (P.), CAMPS (V.), CROISE (F.), GIRAUDET (E.), GIRAUdeT (J.), LAFAYE (J.-Y.), ROUAN (M.), TISSOT (C.), LE TIXERANT (M.), 2002. - Contribution méthodologique pour le couplage de modèle qualitatifs et quantitatifs au sein de Systèmes d'Information Géographique, GDR SIGMA/PEVS/CNRS, 72 p.

FERBER (J.), 1995. - Les systèmes multi-agents ; vers une intelligence collective, Paris, InterÉditions, 544 p.

Gould (S. J.), 1987. - Time's Arrow, Time's Cycle: Myth and Metaphor in the Discovery of Geological Time, Cambridge, Harvard University Press, $222 \mathrm{p}$.

Jensen (C. S.), Clifford (J.), Elmasri (R.), Gadia (S. K.), Hayes (P. J.), Jajodia (S.), 1994. - « A consensus glossary of temporal database concepts ", Sigmod Record, n² 23/1, p. 52-64.

Jordão (L.), Antunes (P.), Videira (N.), Santos (R.), Martinho (S.), Pires (P.), 1996. - « GIS Based Decision Support for ICZM : Costa Vicentina Case Study ", Conference on Socio-Economic Benefits of Integrated Coastal Zone Management, Bremen, $16 \mathrm{p}$.

Klemas (V. V.), Gantt (R. G.), Hassan (H.), Patience (N.), Weatherbee (O. P.), 1995. - Environmental information systems for coastal zone management, $\mathrm{n}^{\circ}$ XIII, World Bank, Washington, $104 \mathrm{p}$.

LEGUAY (J.-M.), 2000. - « Les temps de l'environnement », dans BARRUÉ-PASTOR (M.), BERTRAND (G.) (éd.), Les temps de l'environnement, Toulouse, Presses universitaires du Mirail, p. 19-32.

LE TIXERANT (M.), 2004. - Dynamique des activités humaines en mer côtière. Application à la Mer d'Iroise, Thèse de Géographie, UBO, Brest, 209 p.

Le Tixerant (M.), Rouan (M.), CuQ (F.), Gourmelon (F.), 2003. - « Simulation of Human Activities Dynamics (DAHU) in marine environment ", CoastGIS '03, Genova, Italy, 10 p.

-, 2005. - « Approche dynamique du déroulement d'activités humaines en mer côtières », soumis à Cybergéo.

MuXART (T.), BLANDIN (P.), FRIEDBERG (C.), 1992. - « Hétérogénéité du temps et de l'espace : niveaux d'organisation et échelles spatio-temporelles », dans JoLLIVET M. (éd.), Sciences de la nature, sciences de la société, Les passeurs de frontières, CNRS, p. 243-258.

PALLEAU (C.), 2004. - Modélisation spatio-temporelle du déroulement des activités de cultures marines, et intégration au sein de la plate-forme de simulation DAHU. Application à la conchyliculture, La Rochelle, DESS Ingénérie des Systèmes d'Information, 77 p.

Parent (C.), SPAcCAPIETRA (S.), ZimAny (E.), 1999. - « Spatio-Temporal Conceptual Models : data structures + space + time ", Advance in GIS, Kansas City, 8 p.

RouAN (M.), 2001. - Contribution à l'analyse et à la conception d'un outil de modélisation des activités humaines en bassin versant côtier, Mémoire de DESS en informatique, Université de La Rochelle, 72 p.

SAnders (L.), Pumain (D.), Mathian (H.), Guérin-Pace (F.) Bura (S.), 1997. - « SiMPOP : a multiagents system for the study of urbanism », Environment and Planning, 24, p. 287-305.

SNOdgrass (R. T.), 1992. - « Temporal Databases », dans CAMPARI (I.), Frank (A.), Fromentini (O.) (ed.), Theories and methods of spatio-temporal reasoning in geographic space, Springer-Verlag, p. 22-64. StALJAnSSENS (M.), 1998. - « ICOMIS - A Modeling Tool for Better Decision Making on Coastal Zone Development », Intercoast network, 29 p.

Stonebraker (M.), Rowe (L.), Hirohama (M.), 1990. « The implementation of POSTGRES », IEEE Transaction of Knowledge and Data Engineering, $\mathrm{n}^{\circ} 2 / 1$, p. 125-142. 
TisSOT (C.), RouAn (M.), CROISE (F.), 2001. - « Modélisation du cycle d'élevage porcin hors-sol : étude du modèle breton ", Journées Cassini : Géomatique et espace rural, Montpellier, p. 177-188.

TissoT (C.), 2003. Modélisation spatio-temporelle d'activités humaines à fort impact environnemental. Application à l'étude des pratiques agricoles intensives dans le département du Finistère, Thèse de géographie, Brest, $227 \mathrm{p}$.

TIssot (C.), CUQ (F.), 2004. - « Apport des SIG pour la modélisation spatio-temporelle d'activités humaines ", Revue Internationale de Géomatique, n 14 1/2004, p. 83-96.

Tissot (C.), Le TiXerant (M.), Gourmelon (F.), Le Berre (I.), 2004. - « Modeling interaction between human activities and coastal zone environment », Littoral 2004, Cambridge Publications, Aberdeen, p. 425-431.

TURNER (R. K.), SUBAK (S. E.), AdGER (W. N.), 1996. - « Pressures, trends and impacts in coastal zones: interactions between socio-economic and natural systems ", Environmental Management, $\mathrm{n}^{\circ}$ 20/2, p. 159-173.

WEISS (G.), 1999. - Multiagent systems. A modern approach to distributed artificial intelligence, Cambridge, MIT Press, $643 \mathrm{p}$.

\section{NOTES}

1. Un agent est assimilé à une entité capable d'agir sur elle-même et sur son environnement, qui réagit à ses transformations et qui possède une représentation partielle de cet environnement (Ferber, 1995).

2. Les Systèmes Multi-Agents permettent de faire coexister des agents aux caractéristiques et aux comportements divergents. L'ensemble du système est considéré comme intelligent, c'est-à-dire doté de capacités d'apprentissage et d'adaptation (Weiss, 1999).

3. Un modèle de réalité correspond à une description systémique de la part de réalité que l'on souhaite analyser.

4. On distingue la modélisation « réaliste », objectif des modèles quantitatifs pour la description de la réalité, de la modélisation «vraisemblable » des activités humaines qui n'a pas l'ambition de décrire la réalité de façon exacte [Cuq et al., 2002].

5. La notion d'activité correspond à la représentation archétypique d'un métier, c'est-à-dire qu'elle synthétise les méthodes de gestion individuelles autour de procédés communs à l'ensemble des individus exerçant le même métier.

6. Modèle invariant contenant un ensemble de descripteurs permettant de qualifier une ou plusieurs activité(s) ayant des procédés de production identiques.

7. Un thread est un processus léger et autonome possédant des fonctions prédéfinies comme la synchronisation avec d'autres threads ou encore la possibilité d'utiliser des sections critiques permettant d'éviter les conflits de partage de sources de données communes. 


\section{RÉSUMÉS}

Cette contribution présente un cadre méthodologique appliqué à la zone côtière permettant de simuler le déroulement d'activités humaines dans l'espace et dans le temps dans la perspective d'évaluer leur impact potentiel sur les milieux concernés.

Cette démarche repose sur la constitution d'une plate-forme de simulation capable de rendre compte de la variabilité du déroulement d'activités à fort impact environnemental en tenant compte des facteurs environnementaux (physiques, biologiques, météorologique), réglementaires et socio-économiques pouvant influencer leur pratique.

This contribution deals with a methodological framework dedicated to long-term changes modelling of the coastal environment under strong human activities influences. Our research is focused on a modeling simulation platform fitted to the analysis of interactions between human activities and environment (Cuq, 2001). It integrates the coupling of quantitative and qualitative models within a Geographic Information System (GIS).

The methodology is based on the development of reality models which integrate spatio-temporal constraints (socio-economic, regulation and meteorology) in order to analyse activities progress and impact. This approach also allows to combine simulation results and analyse spatio-temporal interactions between different activities, especially in the aim to highlight strategicals or interests conflicts zones between concurentials activities.

\section{INDEX}

Keywords : coastal zone, human activities, modeling, multi-agent system, GIS

Mots-clés : activité humaine, interactions homme/milieu, modélisation, systèmes multi-agents (SMA), SIG

\section{AUTEURS}

\section{CYRIL TISSOT}

IUEM (Plouzané), GEomer, UMR 6554 LETG - CNRS

\section{MATTHIEU LE TIXERANT}

IUEM (Plouzané), GEomer, UMR 6554 LETG - CNRS

MATHIAS ROUAN

IUEM (Plouzané), GEomer, UMR 6554 LETG - CNRS 